\section{El Código Sanitario Panamericano: hacia una política de salud continental}

\author{
Gregorio Delgado García, ${ }^{1}$ \\ Eduardo Estrella ${ }^{2}$ y Judith Navarro ${ }^{3}$
}

En octubre de 1999 se cumplieron 75 años de la adopción del Código Sanitario Panamericano, primer tratado de sanidad internacional en ser firmado por todas las naciones del continente americano. Este Código, que fue ratificado en La Habana, Cuba, en 1924, en ocasión de la Séptima Conferencia Sanitaria Panamericana, subrayó la contribución de la salud humana al progreso económico de los países y al intercambio comercial $y$, simultáneamente, señaló el papel fundamental de la higiene portuaria en la promoción de la salud. En él se hacía un llamado a la cooperación entre países con la doble finalidad de fortalecer las relaciones comerciales y proteger la salud pública, tareas cuya orquestación fue delegada a la Organización Panamericana de la Salud (OPS).

En honor de tan insigne acontecimiento, el Ministerio de Salud Pública de Cuba, que también cumplía para esas fechas su 90. aniversario, celebró en La Habana del 18 al 22 de octubre una serie de actividades conmemorativas donde se insistió en la necesidad de un esfuerzo mancomunado por encontrar solución a los problemas de orden sanitario y socioeconómico que aquejan hoy en día a los países de la Región. Destacaron entre ellas una Reunión de Ministros de Salud de Iberoamérica, actividad que dio inicio a la semana conmemorativa; la II Conferencia para la Movilización de Recursos Multisectoriales, en la que participaron las autoridades cubanas de salud pública y de otros ministerios, la OPS y otras agencias de las $\mathrm{Na}$ ciones Unidas; y el III Seminario sobre Atención Primaria, que se centró en el intercambio de experiencias en este campo en relación con la reforma del sector sanitario y de la meta de Salud para todos en el siglo $X X I$. Durante la semana de actividades se repasaron los sucesos históricos que llevaron a la firma del Código Sanitario y la influencia que éste ejerció en el control y la prevención de las enfermedades que prevalecían en aquel entonces en Cuba y en los demás países del continente.

La Revista Panamericana de Salud Pública/Pan American Journal of Public Health publica el siguiente relato en reconocimiento de uno de los sucesos de mayor trascendencia en la historia de la salud pública de los países americanos.

\section{ANTECEDENTES}

El Código Sanitario Panamericano fue firmado ad referendum por 18 países de las Américas en el Salón de Actos de la antigua Academia de Ciencias Médicas, Físicas y Naturales —actual Museo de Historia de las Ciencias Dr. Carlos J. Finlay- en La Habana, Cuba, el 14 de noviembre de 
1924 durante la Séptima Conferencia Sanitaria Panamericana. Posteriormente ratificado por todas las repúblicas americanas, y aún vigente hoy en día, el Código representa el mayor logro de la normalización de la salubridad en el continente americano y la culminación de décadas de iniciativas internacionales dirigidas a prolongar la vida y a lograr la felicidad humana.

Las barreras que más obstaculizaban el progreso hacia estas metas sociales eran las enfermedades contagiosas. Para contrarrestarlas, las naciones habían venido imponiendo la práctica de la cuarentena, modelo médico-político utilizado en la Edad Media cuyos comienzos documentados se remontan al siglo XIV y a la administración del puerto de Venecia. Frente a la amenaza de la peste o de una epidemia violenta, se aplicó este "reglamento de urgencia" no solo para controlar los navíos que llegaban a puerto, sino también en las ciudades para detener la extensión de la enfermedad, vigilar a los enfermos, proteger a los sanos, desinfectar las casas con perfumes e inciensos y enterrar a los muertos; la exclusión de los leprosos y su expulsión de las ciudades también formó parte sustancial de este modelo.

Con la expansión colonial europea, el desarrollo de las comunicaciones y la posible propagación de enfermedades exóticas, la cuarentena se convirtió en un arma sanitarista indispensable, pero al mismo tiempo contraproducente para el comercio y la economía. De ahí surgió la necesidad de una ciencia médica positivista, de una salubridad y de una higiene que disminuyeran los tiempos de cuarentena de barcos y puertos y, de ser posible, que eliminaran este modelo por antieconómico. Así lo entendieron las autoridades europeas de las nacientes economías industriales, que en el siglo XIX empezaron a establecer normas sanitarias nacionales y lanzaron un movimiento de cooperación sanitaria internacional.

Sin duda, la epidemia de cólera que en 1848 azotó Europa fue el mejor aliado de los higienistas ingleses, quienes, ante el horrible espectáculo de la muerte de 54000 personas, clamaron por un orden. Inglaterra inició reformas sanitarias basadas en la colección de datos demográficos y de salud, estableció puestos de salud, promulgó políticas sanitarias, llevó a cabo estudios sobre las causas de las enfermedades y, sobre la base de sus conclusiones, aplicó medidas de prevención y control. Así nacieron en Inglaterra la Ley de Salud Pública y la Junta General de Salud, que conforman hitos importantísimos en la historia de la salud pública.

Siguiendo el ejemplo inglés, para imponer un orden interno Alemania y Francia basaron su movimiento sanitario en la creación de organismos de salud pública; la aprobación de normas y códigos; el reciente concepto de policía médica para la vigilancia y control de la población; la aplicación de los nuevos esquemas de medicina preventiva; la utilización de la estadística como base de la higiene pública, y la constitución de esta última como disciplina científica.

Con la correspondiente repercusión en sus colonias de América, desde finales del siglo XVIII España había reformado su ya obsoleta organización de salud pública, centrada en la institución del Real Tribunal del Protomedicato creada en 1477 y trasladada oficialmente a América (México y Perú) en 1570, y la había transformado en las Reales Juntas Superiores Gobernativas de Medicina, de Cirugía y de Farmacia, a las que agregaría a principios del siglo XIX las Reales Juntas de Vacunación de Sanidad y de Beneficencia y Caridad con diferentes niveles administrativos.

No obstante, las amenazas del cólera, de la fiebre amarilla y de la peste bubónica rebasaban las fronteras y era indispensable internacionalizar los problemas de salud pública y configurar un orden externo, supranacional y vinculante. De esa manera surgió lo que vendría a ser una serie de conferencias sanitarias internacionales que tenían dos objetivos fundamentales: suprimir los obstáculos que se oponían al comercio y a los transportes, y defender a Europa y a los estados civilizados de las pestilencias exóticas.

La tradición sanitaria inglesa tuvo una notable influencia en la organización de la salud pública de los Estados Unidos. Ya en las primeras décadas del siglo XIX se había reglamentado la existencia de un Inspector de Salud en las principales ciudades. Esta instancia se ocupaba de la administración sanitaria, el control de epidemias y la recogida y elaboración de datos estadísticos. Las epidemias de cólera de 1830 y 1849 causaron la muerte de cientos de miles de personas. Solamente en la ciudad de Nueva Orleans, en el estado de Luisiana, murieron 8000 de los 55000 habitantes. Los distintos estados del país hicieron frente a la amenaza de estas enfermedades con el establecimiento de departamentos de salud: en Luisiana en 1855, en Massachusetts en 1869 y en California en 1870. En 1872 se creó la Asociación Estadounidense de Salud Pública y en 1879 la Junta Nacional de Salud. A partir de 1878 se estableció la cuarentena oficial en los puertos de los Estados Unidos como resultado directo de la cuarta pandemia de cólera, que se inició en la India en 1863, pasó rápidamente a Europa a través del Mar Rojo y de los peregrinajes a La Meca, e invadió amplias zonas de América. En 1883 el Cirujano General del Servicio de Hospitales Navales se hizo responsable de la cuarentena interestadual e internacional (en 1912 el nombre de este servicio cambió al de Servicio de Salud Pública de los Estados Unidos, que tanta importancia tendría en el desarrollo de la Oficina Sanitaria Panamericana). 
Participantes en la Séptima Conferencia Sanitaria Panamericana (La Habana, Cuba, 1924), en la que se redactó el primer tratado firmado por todas las Repúblicas Americanas: el Código Sanitario Panamericano, que sigue en vigor hoy en día.

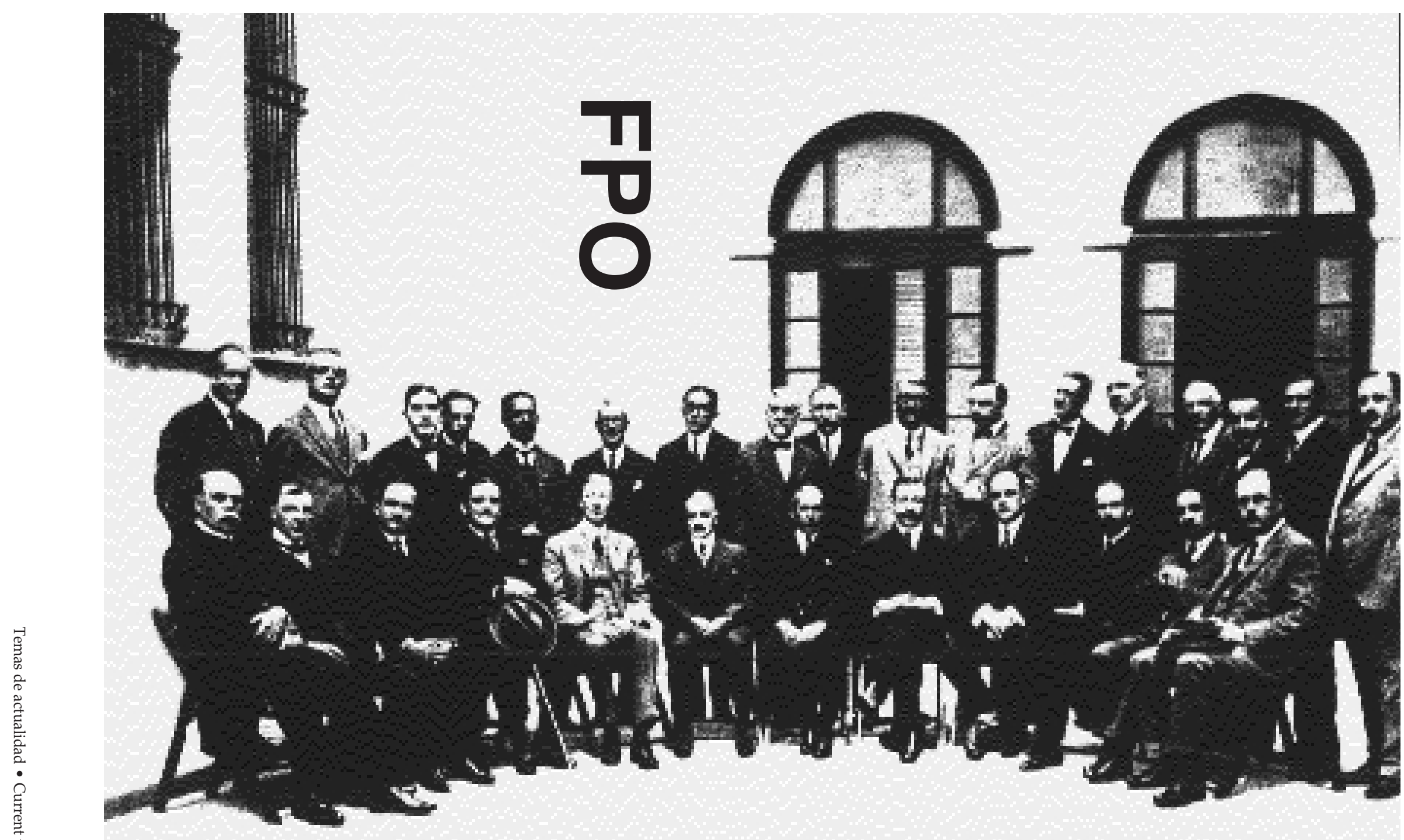


Por otra parte, en la segunda mitad del siglo XIX, los Estados Unidos se colocaron al frente del desarrollo del capitalismo y su influencia en los otros países americanos es muy notable. Estos últimos se convierten en economías productoras de materias primas y exportadoras de productos agrícolas dependientes del capital internacional. Los puertos de estos países eran los núcleos de estas relaciones comerciales, ya que en ellos se acumulan las riquezas provenientes del interior en su paso hacia otros países. De su buen funcionamiento depende que se mantenga o no el flujo de productos y la extracción de las riquezas naturales. Por esta razón, el puerto constituye el objetivo básico de la acción sanitaria. El saneamiento de los puertos y de los sitios de producción constituía, entonces, una necesidad fundamental para el desarrollo económico en las Américas, necesidad apremiante, cuya resolución era constantemente reclamada por la presencia de epidemias de fiebre amarilla, peste bubónica y cólera y la perpetua amenaza de la malaria.

En este marco, sobre el sustento de la tradición sanitaria de los Estados Unidos y de la participación creciente de un movimiento sanitario propio de los países latinoamericanos, encabezado por los sanitaristas cubanos, y a fin de resolver un problema crítico que afectaba al comercio y a la libre circulación de mercancías, se creó un orden para las Américas, el movimiento sanitarista panamericano; una institución conocida por la Oficina Sanitaria Panamericana, y un tratado internacional llamado el Código Sanitario Panamericano. En el nacimiento de este orden, de esta institución y de este código prevalecía también, en el fondo, una preocupación ética por la salud de la población.

\section{CONFERENCIAS INTERNACIONALES EN PRO DE LA SALUD}

Se convocó la I Conferencia Sanitaria Internacional en París del 23 de junio de 1851 al 19 de enero de 1852. Este primer intento de internacionalizar los problemas de la salud pública contó con la asistencia de doce países —once europeos y uno asiáticointeresados en llegar a un acuerdo sobre las condiciones mínimas de la cuarentena marítima y en prestar, de ese modo, importantes servicios al comercio y a la navegación en el Mediterráneo, así como en proteger la salud pública. Cada país estuvo representado por dos delegados, uno médico y el otro, funcionario diplomático. Después de celebrar 48 sesiones plenarias y numerosas reuniones de sus comisiones, se aprobó un convenio sanitario internacional que comprendía 137 artículos. El 19 de diciembre de 1851, los representantes de las 12 naciones firmaron el proyecto de convenio y el 16 de enero siguiente, un proyecto revisado. Sin embargo, las firmas de los representantes no obligaban a sus respectivos gobiernos, y cuatro meses más tarde sólo lo habían firmado cinco países; aún así, esas firmas necesitaban ser ratificadas. El 18 de mayo de 1852, Cerdeña y Francia intercambiaron instrumentos de ratificación y el convenio entró en vigor entre ambos países. Portugal se sumó más tarde. No obstante, en 1865 este país y Cerdeña se retiraron y el convenio quedó sin efecto alguno.

La II Conferencia Sanitaria Internacional, en la que no participaron delegados médicos, se reunió en 1859, también en París, y duró cinco meses. Se aprobó en ella, por la mayoría de los delegados, un nuevo proyecto de convenio sanitario internacional, del cual no se tuvo después noticia alguna. La III Conferencia, celebrada en Constantinopla en 1866, tampoco dejó huellas duraderas.

El rasgo más importante de la IV Conferencia Sanitaria Internacional, celebrada en Viena durante todo el mes de junio de 1874, fue la presentación por la delegación de Francia de un proyecto para establecer una Comisión Internacional Permanente de las Epidemias, con sede en Viena, el cual se aprobó por unanimidad. La Comisión había de componerse de médicos designados por los gobiernos participantes y sus principales objetivos serían estudiar la etiología y la profilaxis del cólera, la epidemiología del cólera en los buques y puertos, el período de incubación de la enfermedad y las lluvias y condiciones telúricas en las regiones del Mediterráneo Oriental y del Mar Negro. Con los resultados de estas investigaciones se redactaría un nuevo código sanitario internacional.

En la V Conferencia Sanitaria Internacional, reunida en Washington, D. C., de enero a marzo de 1881 - primera que se celebraba en el continente occidental y con la asistencia, por primera vez, de países americanos- el delegado de Austria-Hungría presentó un proyecto para la institución de dos agencias internacionales permanentes de avisos sanitarios que se establecerían en Viena y en La Habana. La primera recogería los informes sanitarios de Europa, Asia y África y la segunda extendería su esfera de acción en el continente americano y las islas que geográficamente le correspondían, salvo las modificaciones que el estado de las comunicaciones telegráficas pudiera hacer necesarias. Aunque fue ampliamente discutido y aprobado, dicho proyecto nunca llegó a entrar en vigor.

En la V Conferencia estuvieron presentes los siguientes países americanos: Argentina, Bolivia, Brasil, Chile, Estados Unidos de América, Haití, México, Perú y Venezuela. Canadá, que era dominio desde 1867, estuvo representado por un delegado especial incluido en la delegación de la Gran 
Bretaña. Aunque eran entonces provincias de España, Cuba y Puerto Rico estuvieron representados por un miembro especial de la delegación española, el doctor Carlos J. Finlay. El hecho más trascendental de esta Conferencia, que lamentablemente pasó casi inadvertida, fue la presentación por Finlay de su teoría de la "tercera condición independiente" para la transmisión de la fiebre amarilla:

Mi opinión personal es que tres condiciones son, en efecto, necesarias para que la fiebre amarilla se propague: 1. La existencia previa de un caso de fiebre amarilla comprendido dentro de ciertos límites de tiempo con respecto al momento actual. 2. La presencia de un sujeto apto para contraer la enfermedad. 3. La presencia de un agente cuya existencia sea completamente independiente de la enfermedad y del enfermo, pero necesaria para transmitir la enfermedad del individuo enfermo al hombre sano.

La presencia de este agente y su "destrucción" o el "alejamiento de las vías por donde se propaga la enfermedad", que Finlay preconizó en esta Conferencia, llegarían a ser los pilares de la acción sanitaria en el siglo XX.

En lo que quedaba del siglo pasado, se siguieron celebrando conferencias sanitarias, de la sexta a la décima: Roma (1885), Venecia (1892), Dresden (1893), París (1894) y Venecia (1897). En cada una de ellas se estableció un convenio internacional de alcance limitado relacionado principalmente con el cólera y la peste. Los objetivos de estos convenios eran fundamentalmente dos: primero, suprimir los obstáculos al comercio y a los transportes y, segundo, proteger a Europa contra las epidemias de enfermedades exóticas.

Independientemente de los resultados de estas conferencias, Argentina, Brasil y Uruguay firmaron en Río de Janeiro, del 25 al 26 de noviembre de 1887, una Convención Sanitaria Internacional. Unos meses antes, el 3 de julio del mismo año, el Gobierno de Perú había invitado a todas las naciones de América a un Congreso Sanitario Americano que se esperaba tuviera lugar en Lima el 1 de noviembre, pero que no se inauguró hasta el 2 de enero de 1888. En ese congreso estuvieron representados solamente Bolivia, Chile, Ecuador y Perú. Sus labores se prolongaron hasta el 12 de marzo, dando como resultado una convención cuyas capitulaciones se refirieron a puntos trascendentales, entre ellos, la profilaxis de la fiebre amarilla.

La Primera Conferencia Internacional Americana efectuada en Washington, D. C., que duró del 2 de octubre de 1889 al 19 de abril de 1890, dio un impulso extraordinario al panamericanismo (y fue el tema principal de 11 crónicas llenas de advertencias políticas por el escritor y patriota cubano José
Martí, que se publicaron en el diario La Nación de Buenos Aires). En la sesión del 7 de diciembre de 1889 sus delegados aprobaron la creación de la $X$ Comisión, compuesta por siete miembros de cinco países (Brasil, Estados Unidos, Nicaragua, Perú y Venezuela), "para tomar en consideración e informar sobre los nuevos métodos de establecer y mantener reglamentos sanitarios en el comercio entre los varios países representados en la Conferencia". La X Comisión dictaminó y la Conferencia recomendó a las repúblicas americanas que adoptaran la Convención Sanitaria Internacional de Río de Janeiro (1887) o el texto de la Convención Sanitaria del Congreso de Lima (1888).

Con el antecedente histórico de estos convenios sanitarios internacionales, el X Comité, el de la Política Sanitaria Internacional, de la Segunda Conferencia Internacional de los Estados Americanos (ciudad de México, 22 de octubre de 1901 al 22 de enero de 1902) presentó un informe en enero de 1902, el cual fue aprobado y en el que se recomendaba que la Oficina Internacional de las Repúblicas Americanas (actual Organización de los Estados Americanos) convocara a una convención general de representantes de las oficinas de salubridad de las repúblicas de América para formular acuerdos y disposiciones sanitarias y celebrar periódicamente convenciones de salud. La convención general también debería designar una junta ejecutiva permanente que se denominaría la Oficina Sanitaria Internacional, que tendría su sede en Washington, D. C.

\section{LA OFICINA SANITARIA INTERNACIONAL Y LAS PRIMERAS CONVENCIONES SANITARIAS PANAMERICANAS}

La Primera Convención Sanitaria Internacional de las Repúblicas Americanas se celebró en Washington, D. C., del 2 al 5 de diciembre de 1902 con la asistencia de 10 países. El 2 de diciembre, día de la inauguración, se fundó la Oficina Sanitaria Internacional, que tenía las siguientes funciones:

a) Solicitar de cada país que se sirva remitir pronta y regularmente a la Oficina todos los datos relativos al estado sanitario de sus puertos y territorio nacional.

b) Obtener toda la ayuda posible para hacer estudios científicos completos de los brotes de enfermedades contagiosas que pudieran ocurrir en los países.

c) Proporcionar su mayor ayuda y su experiencia a fin de obtener la mejor protección posible para la salud pública de los países a fin de conseguir la eliminación de la enfermedad y facilitar el comercio entre las naciones. 
d) Estimular, ayudar o imponer todos los medios jurídicos a su disposición para el saneamiento de los puertos marítimos, incluyendo la introducción de mejoras sanitarias en la rada, el sistema de avenamiento, el drenaje del suelo, la pavimentación y la eliminación de la infección de los edificios, así como la destrucción de mosquitos y otros insectos nocivos.

La primera época de la Oficina Sanitaria Internacional fue muy importante, pero tuvo una actividad limitada y se centró básicamente en el tema de la internacionalización de los problemas sanitarios y en la formulación de recomendaciones. Se convocaron varias Convenciones Sanitarias Internacionales, denominadas posteriormente Conferencias Sanitarias Panamericanas, en las cuales los delegados de los países miembros aprobaban e impulsaban las propuestas de los responsables de la Oficina.

Entre las obligaciones asumidas por los países al asociarse con la nueva Oficina Sanitaria Internacional destacan proveer información sobre las condiciones sanitarias, reducir la cuarentena a los períodos más cortos posibles que fueran compatibles con la seguridad pública y los conocimientos científicos, y llevar a cabo el saneamiento de los puertos. Para cumplir con este último objetivo, se puso en práctica el método para la profilaxis de la fiebre amarilla enunciado por Finlay en la Conferencia de Washington de 1881 respecto a "la tercera condición independiente". Esta tercera condición había sido descrita claramente, unos meses después, en la sesión de la Academia de Ciencias de La Habana el 14 de agosto de 1881, cuando Finlay presentó su trabajo "El mosquito hipotéticamente considerado como agente de transmisión de la fiebre amarilla". Las investigaciones experimentales realizadas con posterioridad por el sabio cubano y por la Cuarta Comisión Estadounidense para el Estudio de la Fiebre Amarilla, dirigida por Walter Reed en 1900, confirmaron la teoría de Finlay, convirtiéndose inmediatamente la lucha contra el mosquito en el núcleo de la nueva acción sanitaria, con beneficios directos para la salud de la población.

$\mathrm{Al}$ año siguiente de creada la Oficina, se inauguró la XI Conferencia Sanitaria Internacional (1903) en París y su principal tarea consistió en unificar los cuatro convenios sanitarios aprobados en conferencias anteriores y refundirlos en un instrumento único, el Convenio Sanitario Internacional de 1903, cuyas disposiciones se referían tanto al cólera como a la peste y la fiebre amarilla. Se trataba de tres enfermedades cuyos modos de transmisión eran totalmente distintos y para las cuales se disponía, por primera vez, de un conjunto de datos científicos aceptados universalmente. El Cirujano General del Servicio de Salud Pública de los Estados Unidos, William C. Gorgas, resumió la labor realizada por la Cuarta Comisión para el Estudio de la Fiebre Amarilla, del Ejército de los Estados Unidos, que confirmó el descubrimiento de Finlay, y la subsiguiente campaña encaminada a erradicar Aedes aegypti de La Habana, que estuvo dirigida por el propio Gorgas. No obstante, el Convenio Sanitario Internacional de París de 1903 no tenía vigencia para las Américas. Por lo tanto, en 1904 las repúblicas de Argentina, Brasil, Paraguay y Uruguay firmaron, en Río de Janeiro, una nueva Convención Sanitaria Internacional, del todo ajena a las actividades de la Oficina Sanitaria Internacional de Washington.

La Segunda Convención Sanitaria Internacional, celebrada también en Washington, D. C., en octubre de 1905, revistió una gran importancia, pues en ella se definió la acción de la Oficina - codificar los procedimientos de sanidad internacional, particularmente en el control de la fiebre amarilla- basada en las medidas aplicadas en Cuba, la Zona del Canal de Panamá y México. Estas medidas fueron tomadas en forma recíproca en dicha Convención y firmadas ad referendum en Washington, D. C., el 14 de octubre de 1905, como primer Código Sanitario Panamericano.

Tal importancia tenía para las Américas este primer Código, que en diciembre de 1907 la Oficina recibió en México la autorización de la Tercera Convención Sanitaria Internacional para establecer relaciones con la recién creada Oficina Internacional de Higiene Pública con sede en París y para recomendar a los países europeos que adoptaran el Código Sanitario de Washington de 1905, por tener en cuenta la necesidad de que las colonias de Francia, Gran Bretaña y los Países Bajos en el hemisferio occidental observaran las resoluciones de las repúblicas americanas sobre la fiebre amarilla.

La Tercera Conferencia Internacional Americana (Río de Janeiro, 21 de junio al 26 de agosto de 1906), ante la dualidad en el continente de dos convenciones sanitarias vigentes, la de Río de Janeiro de 1904 y la de Washington de 1905, estudió a ambas y se adhirió al primer Código Sanitario Panamericano firmado en Washington, D. C. en 1905. Unos años más tarde, la Cuarta Conferencia Internacional Americana (Buenos Aires, 12 de julio al 30 de agosto de 1910) recomendó la adopción de dicho Código a aquellos gobiernos de América que no lo habían adoptado todavía.

Una lectura de la correspondencia del Cirujano General del Servicio de Salud Pública de los Estados Unidos durante el período de 1906 a 1923 revela que muchos países habían firmado convenciones sanitarias bilaterales y posteriormente multilaterales (Río de Janeiro y Lima). Informes enviados al Secretario del Tesoro de los Estados Unidos por 
cónsules de ese país, destinados en ciudades latinoamericanas, describían las condiciones sanitarias del continente, sobre todo las de los puertos, citaban estadísticas de mortalidad, y notificaban las enfermedades principales, que eran la fiebre amarilla, la peste, el cólera, y la viruela. Describían asimismo los códigos de sanidad en vigencia y otras medidas sanitarias: regulaciones, decretos, desratización, desinfección y fumigación de buques, legislación, publicación de boletines epidemiológicos nacionales, y esfuerzos de la comunidad para mejorar los servicios locales de salud.

Con el transcurso de los años, las posibilidades de ejercer una acción más dinámica aumentaron y la Oficina pudo aplicar con mayor eficacia las medidas de control y erradicación de la fiebre amarilla, la peste bubónica y la malaria. Aunque en la Cuarta Conferencia Sanitaria, realizada en San José en 1909, persistía el interés por las enfermedades transmisibles y sujetas a cuarentena, el ámbito de acción se extendió a la salud en general, con el planteamiento de temas como los siguientes:

la vacunación obligatoria contra la viruela; las campañas antipalúdica y antituberculosa, la centralización de la legislación sanitaria nacional; el impulso al estudio de las enfermedades tropicales para darles base científica y mayor auge a la parasitología y a la anatomía patológica; el establecimiento de laboratorios en los puertos no solo para fines de diagnóstico sino para hacer investigaciones originales en medicina tropical y patología general, siguiendo las orientaciones que las autoridades sanitarias juzgaran adecuadas.

En la Quinta Conferencia Sanitaria, reunida en Santiago de Chile en 1911, se recomendó a los gobiernos la formación de especialistas calificados en salud pública "mediante cursos formales y prácticas de higiene y saneamiento". También se expresó, por primera vez, la conveniencia de conformar comisiones de información sanitaria para comunicar a los gobiernos las obligaciones impuestas por las conferencias. En apenas 10 años de vida institucional, esta labor se había convertido en un combate de alcance hemisférico contra las enfermedades y las pérdidas humanas y económicas consiguientes.

Debido a la Primera Guerra Mundial, la Sexta Conferencia Sanitaria, programada para 1915, se postergó hasta 1920, cuando se celebró del 12 al 20 de diciembre en Montevideo. Entre sus resoluciones se destacaba la de fundar y editar un "Boletín Internacional de las Repúblicas Americanas" — después conocido como Boletín de la Oficina Sanitaria Panamericana y hoy en día publicado como Revista Panamericana de Salud Pública- concebido como el medio más eficaz para difundir la información sani- taria y los avances científicos en el campo de la salud pública. Esta conferencia también autorizó una reorganización de la Oficina, dándole mayores posibilidades para llevar a cabo sus actividades.

A la joven organización le esperaban nuevos desafíos y responsabilidades. El primer artículo del primer número del Boletín, publicado en mayo de 1922, por J. H. White, se tituló "La importancia de la cooperación sanitaria entre las naciones" y describe la razón de ser de la Oficina:

Esta Oficina hace los mayores esfuerzos para mejorar todo lo que se relaciona con las condiciones higiénicas y el saneamiento, mejorar también el servicio de cuarentena y dominar e impedir la propagación de enfermedades contagiosas que deben ser denunciadas por los médicos, para eliminarlas y facilitar de esta manera el comercio entre las repúblicas del Hemisferio Occidental. [Su objetivo es] lograr una inteligencia que acabará por producir la uniformidad en cuanto a procedimientos, sobre todo en lo que atañe al manejo del comercio marítimo y la uniformidad en la aplicación de los métodos para dominar las enfermedades contagiosas.

Vislumbra la adopción de un nuevo instrumento de orden, el establecimiento de un código panamericano, ante la amenaza de las enfermedades contagiosas:

Ha llegado el momento en que debe establecerse una inteligencia y cooperación más intimas entre las autoridades sanitarias de las Américas, en su incesante lucha contra la propagación de las enfermedades transmisibles con el fin de obtener la completa eliminación de las mismas. Esta cooperación debe también hacerse extensiva al establecimiento de un reglamento sanitario que comprende la adopción de reglas seguras y razonadas que rijan la cuarentena de los viajeros y la importación y exportación de carga en el canje comercial panamericano.

Y haciendo eco de los tratados internacionales que se venían celebrando desde mediados del siglo XIX en lo que se refiere a la estrecha relación entre comercio y salud, afirma:

Si todos y cada uno metemos el hombro y cooperamos en seguida, esto puede hacerse y se hará, debiendo agregarse que no puede concebirse hazaña más noble que la verdadera cooperación sanitaria panamericana [cuyo objetivo es] . . . la magna obra de salvar vidas humanas, y la no menos importante de hacer desaparecer obstáculos innecesarios en las transacciones comerciales [pues] el que estimula y fomenta condiciones de seguridad para el comercio a la vez estimula la salud y la felicidad para su pueblo. 


\section{LA SÉPTIMA CONFERENCIA SANITARIA PANAMERICANA Y EL CÓDIGO}

El proceso de institucionalizar e internacionalizar la salud pública estaba incompleto. Si bien se tenía una Oficina responsable y las conferencias habían establecido normas, faltaba un instrumento político-científico que obligara a los países a cumplir ciertos fines para responder a los desafíos de un mundo cada vez más interdependiente, más intercomunicado y con mayor intercambio comercial.

Ante la conciencia colectiva de esta necesidad, la Quinta Conferencia Internacional Americana, reunida en Santiago de Chile del 25 de marzo al 13 de mayo de 1923, decidió que la Oficina Sanitaria Internacional se encargara de preparar un proyecto de Código de Ley Marítima Internacional y que el proyecto fuera estudiado y adoptado en forma de tratado por la Séptima Conferencia Sanitaria Internacional, que se efectuaría en La Habana en fecha fijada durante el año 1923. La Quinta Conferencia Internacional especificó que la Séptima Conferencia Sanitaria estudiara detenidamente las siguientes proposiciones:

1. Debe considerarse infectada una embarcación en la cual se constate la presencia de ratas pestosas, aunque durante el viaje no se produzca ningún caso humano de peste;

2. La rehabilitación de una localidad infectada por la peste solo debe hacerse efectiva treinta días después de constatada la existencia de ratas pestosas y de puestas en práctica todas las medidas más rigurosas de desinfección;

3. Los navíos portadores de enfermos de fiebre amarilla no deben ser considerados como infectados;

4. El plazo para las medidas sanitarias en los casos de fiebre amarilla debe ser de trece días;

5. En las embarcaciones procedentes de puertos contaminados por el cólera debe hacerse sistemáticamente un examen bacteriológico de los pasajeros y los tripulantes;

6. Debe ser obligatoria la permanencia de los inspectores sanitarios a bordo de las embarcaciones que se dirijan a los puertos americanos;

7. Debe incluirse el tifus exantemático entre las enfermedades de notificación obligatoria;

8. Debe crearse una Sub-Comisión de Higiene en cualquiera capital suramericana, para el estudio de las cuestiones sanitarias de interés común a los diferentes países. Dicha Sub-Comisión estará constituida por higienistas de cada país y sus decisiones serán sometidas, antes de ser aprobadas, a la consideración de la Comisión Central de Higiene de Washington.

Igualmente resolvió esta Quinta Conferencia Internacional que "La Oficina Sanitaria Internacio- nal sea conocida y designada como Oficina Sanitaria Panamericana y que las Conferencias Sanitarias Internacionales [que habían adoptado este nombre en 1901] en adelante sean conocidas y designadas como Conferencias Sanitarias Panamericanas".

Al convocar la Séptima Conferencia Sanitaria, L. S. Rowe, Director General de la Unión Panamericana, invitó a los gobiernos de los Estados Americanos a que asistieran a ella, anunciando que "Cierto número de importantes cuestiones sanitarias será discutido en esta Conferencia, y se espera que todas las naciones interesadas estarán debidamente representadas."

La Séptima Conferencia Sanitaria Panamericana se reunió en La Habana del 5 al 15 de noviembre de 1924, con delegados de 18 repúblicas: Argentina (2), Brasil (2), Chile (1), Colombia (1), Costa Rica (1), Cuba (6), El Salvador (1), Estados Unidos de América (3), Guatemala (1), Haití (1), Honduras (1), México (1), Panamá (1), Paraguay (1), Perú (1), República Dominicana (1), Uruguay (1) y Venezuela (2) —en total, 28 delegados. Faltaron Bolivia, Ecuador y Nicaragua. Además de los seis delegados de Cuba, eran también de ese país los de Costa Rica, Guatemala y Honduras, y el de Colombia, aunque nacido en su país, llevaba casi medio siglo de ejercicio médico en La Habana. También estaban representadas la Oficina Sanitaria Panamericana y la Sección de Higiene de la Liga de las Naciones.

El discurso inaugural del Secretario de Estado de la República de Cuba, doctor Carlos Manuel de Céspedes y de Quesada, subrayó la importancia histórica del panamericanismo, desde la Asamblea General de Plenipotenciarios, convocado por Simón Bolívar en 1825, hasta la Quinta Conferencia Sanitaria Internacional en 1881, cuando es "reconocida la evidencia de que es indispensable la cooperación sanitaria para el desarrollo del comercio internacional", y afirmó que "Esta Séptima Conferencia Sanitaria Panamericana, que se halla reunida ahora, promete ser la más importante de todas. Ella afrontará con ánimo resuelto la magna tarea de forjar el Código Sanitario Marítimo Panamericano."

Presidió la Comisión Ejecutiva de la Conferencia el doctor Mario García Lebredo (Cuba), posteriormente Director de Honor de la Oficina Sanitaria Panamericana; sirvieron de Vocales, los doctores Hugh S. Cumming (E.U.A.), Director de la Oficina Sanitaria Panamericana y Cirujano General Jefe de Servicios de Salud Pública de los Estados Unidos, Nascimento Gurgel (Brasil), Joaquín Llambías (Argentina), Alfonso Pruneda (México) y Carlos Enrique Paz Soldán (Perú), todas figuras ilustres de la salud pública en América.

El estudio del documento fue encargado a la Comisión del Código de Sanidad Marítima Pan- 
americano, presidida por el profesor doctor Gregorio Aráoz Alfaro (Argentina), personalidad insigne de la semiología y la pediatría latinoamericanas; fue Secretario el doctor Jaime de la Guardia (Panamá), destacado cirujano, graduado del Jefferson Medical College de Filadelfia y la Universidad de La Habana; y sirvieron de Vocales los doctores Raúl Almeida Magalhães (Brasil), Secretario General del Departamento de Salud Pública de Brasil; Enrique Tejera (Venezuela), Jefe del Laboratorio de Microbiología de la Dirección de Sanidad de Venezuela; Hugo Roberts Fernández (Cuba), uno de los fundadores de la organización de la sanidad cubana, creador del Servicio de Cuarentena en 1902 y General de la Brigada del Ejército Libertador de Cuba; Richard H. Creel (E.U.A.), Jefe de Cuarentenas del Puerto de San Francisco, California; Romano Pérez Cabral (República Dominicana), profesor de la Universidad de Santo Domingo; Carlos E. Paz Soldán (Perú), sanitarista de prestigio internacional, profesor de Higiene de la Universidad de San Marcos de Lima y autor de más de 600 trabajos científicos; José Varela Zequeira (Costa Rica), cubano, destacado hombre de letras y eminente profesor de Anatomía de la Universidad de La Habana; José de Cubas Serrate (Guatemala), destacado cirujano cubano; Arístides Agramonte Simoni (Honduras), bacteriólogo y sanitarista cubano de prestigio internacional, nominado con el doctor Carlos J. Finlay para el premio Nobel de Medicina y Fisiología; Leopoldo B. Paz (El Salvador), Director General de Sanidad y Presidente de la Asamblea Nacional Legislativa de El Salvador; Justo F. González (Uruguay), profesor de Higiene de la Universidad de Montevideo; Andrés Gubetich (Paraguay), profesor de la Facultad de Medicina de la Universidad Nacional de Asunción; Alfonso Pruneda (México), prestigioso profesor de Patología General de la Escuela Nacional de Medicina de México; Charles Mathon (Haití), destacado profesor de Clínica Médica de la Universidad de Haití; Ricardo Gutiérrez Lee (Colombia), Embajador Plenipotenciario en Cuba, con casi cinco décadas de ejercicio brillante de la medicina en La Habana y John D. Long (Oficina Sanitaria Panamericana), higienista eminente, Cirujano General Auxiliar del Servicio de Salud Pública de los Estados Unidos y Auxiliar del Director de la Oficina Sanitaria Panamericana.

La Comisión estudió el proyecto de Código de Ley Marítima Internacional, posteriormente aprobado y adoptado en forma de tratado o Código Sanitario Panamericano por la Conferencia, el cual consta de 13 capítulos y 63 artículos.

El Capítulo I lo constituyen dos artículos. En el primero se encuentran los objetivos del Código, que son:
1. Prevenir la propagación internacional de infecciones o enfermedades susceptibles de trasmitirse a seres humanos.

2. Estimular o adoptar medidas cooperativas encaminadas a impedir la introducción y propagación de enfermedades en los territorios de los gobiernos signatarios o procedentes de los mismos.

3. Uniformar la recolección de datos estadísticos relativos a la morbilidad y mortalidad en los países de los gobiernos signatarios.

4. Estimular el intercambio de informes que puedan ser valiosos para mejorar la sanidad pública y combatir las enfermedades propias del hombre.

5. Uniformar las medidas empleadas en los lugares de entrada para impedir la introducción de enfermedades transmisibles propias del hombre, a fin de que pueda obtenerse mayor protección contra ellas y eliminarse toda barrera o estorbo innecesario para el comercio y la comunicación internacional.

El segundo artículo del Capítulo I está destinado a las definiciones de 11 términos contenidos en el Código.

El Capítulo II lo forman tres secciones en las que se tratan notificaciones e informes ulteriores a otros países (6 artículos), la publicación de las medidas preventivas (3) y las estadísticas de morbilidad y mortalidad (4).

El Capítulo III está dedicado a documentos sanitarios y consta de dos secciones: la primera se refiere a patentes de sanidad (11 artículos) y la segunda, a otros documentos (2).

Los Capítulos IV a VIII comprenden diversos aspectos tales como: la clasificación de los puertos (6 artículos), la clasificación de los buques (3), el tratamiento de los buques (10), los modelos de fumigación (3) y los médicos de los buques (3).

El Capítulo IX es de gran importancia, pues está dedicado a la Oficina Sanitaria Panamericana, sus funciones y deberes. En su primer artículo se especifica "que la organización, funciones y deberes de la Oficina Sanitaria Panamericana deberán incluir aquello que hasta ahora han dispuesto o determinado las varias conferencias sanitarias internacionales y otras conferencias de las Repúblicas Americanas y también las funciones y deberes administrativos adicionales que en lo sucesivo dispongan o prescriban las Conferencias Sanitarias Panamericanas". Otros seis artículos completan el capítulo.

El Capítulo X comprende un artículo dedicado a naves aéreas.

El Capítulo XI declara en su único artículo que "Excepción hecha de los casos que estén en con- 
flicto con las prescripciones de la presente Convención, continuarán en todas sus fuerzas y vigor los artículos 5, 6, 13, 14, 15, 16, 17, 18, 25, 30, 32, 33, 34, $37,38,39,40,41,42,43,44,45,49$ y 50 de la II Convención Sanitaria Panamericana celebrada en Washington, D. C. el 14 de octubre de 1905 o primer Código Sanitario".

El Capítulo XII aclara que "Se tiene por entendido que el presente Código no anula ni altera la validez o fuerza de ningún tratado, convención o acuerdo que exista entre algunos de los gobiernos signatarios y cualquier otro gobierno".

El Capítulo XIII, final, dedica su único artículo a disposiciones transitorias.

El Código fue adoptado ad referendum y firmado en la Ciudad de La Habana por los delegados de los 18 países presentes en la Séptima Conferencia Panamericana el día 14 de noviembre de 1924, en dos ejemplares originales, en inglés y español respectivamente, los cuales se depositaron en la Secretaría de Estado de la República de Cuba a fin de que pudieran sacarse copias certificadas de él, tanto en inglés como en español, para ser remitidas por la vía diplomática a cada uno de los gobiernos signatarios.

Con el propósito de seguir las buenas normas de la profilaxis, el Código buscaba: "prevenir la propagación internacional de infecciones o enfermedades susceptibles de transmitirse a seres humanos" y, en el caso de que éstas surgieran, "adoptar medidas cooperativas para impedir la introducción y propagación" a otros territorios y detenerlas en sus lugares de gestación. Se necesitaba buena información, un intercambio rápido de datos estadísticos uniformados y comparables y, por supuesto, una homologación de "las medidas empleadas en los lugares de entrada para impedir la transmisión". Esto último, según un comentario editorial publicado en el Boletín de la Oficina Sanitaria Panamericana en 1929, "fue una decisión de incalculable importancia, ya que todo rechazo o prolongada detención de las exportaciones de un país en donde reine una enfermedad cuarentenable ocasiona naturalmente disputas, roces y hasta enemistad". Al respecto, algo que llama la atención en el Código es la "Clasificación de los Puertos" en las categorías de infectado, sospechoso, limpio clase A, limpio clase B y no clasificado. Esta tipología suscitó una competencia entre los países para que cada uno de sus puertos adquiriera el título de "limpio clase A".

El Código cubría, además, un campo mucho más amplio que los tratados anteriores: fue más específico y definido en cuanto a sus estipulaciones con respecto a buques, puertos y períodos de cuarentena; abarcaba, por primera vez (y dando lugar a la eliminación de toda referencia a lo "marítimo"), aeronaves; añadió previsiones con respecto a un número importante de temas, incluidas las estadísticas vitales y las posibilidades de cooperación interamericana en la salud y campos afines, y asignó funciones y responsabilidades más amplias a la Oficina Sanitaria Panamericana como organismo coordinador central de las actividades de salud internacional en las Américas.

\section{RATIFICACIONES Y ENMIENDAS POSTERIORES DEL CÓDIGO}

Depositado oficialmente en el Gobierno de Cuba el 13 de abril de 1925, entre las primeras ratificaciones se recibieron la del Senado de Cuba y la del Senado de los Estados Unidos de América. Fue ratificado además en poco tiempo, por Chile, Costa Rica, Honduras y Perú. Nicaragua declaró su adhesión por no haber sido país signatario.

En la Primera Conferencia de Directores de Sanidad de las Repúblicas Americanas desarrollada en Washington, D. C., del 28 al 30 de septiembre de 1926, se estudió la interpretación de varios artículos del Código y en la Octava Conferencia Sanitaria Panamericana, celebrada en Lima del 12 al 20 de octubre de 1927, se aprobó ad referendum un Protocolo Adicional al Código Sanitario Panamericano en el que se dice:

Las ratificaciones del Código Sanitario se depositarán en la Secretaría de Estado de la República de Cuba; y el Gobierno cubano comunicará esas ratificaciones a los demás Estados Signatarios, comunicación que producirá el efecto del canje de ratificaciones. La convención empezará a regir en cada uno de los Estados Signatarios en la fecha de la ratificación por dicho Estado, y quedará en vigencia sin limitación de tiempo, reservándose cada uno de los Estados Signatarios o Adheridos el derecho de retirarse de la convención mediante aviso dado en debida forma al Gobierno de la República de Cuba, con un año de anticipación.

La ratificación del Código Sanitario Panamericano fue tema de la Sexta Conferencia Internacional Americana, la cual resolvió, el 3 de febrero de 1928:

Recomendar a todos los países que no han ratificado hasta la fecha el Código Sanitario Panamericano [.. . ] que procuren a la mayor brevedad posible ratificarlo y aplicar sus disposiciones en su mayor amplitud, a efecto de que, todos y cada uno de los países de la Unión Panamericana, estén en aptitud de presentar en la próxima Novena Conferencia Sanitaria Panamericana, que habrá de efectuarse en Buenos Aires, las observaciones que sus respectivas experiencias 
les hubieren ofrecido y poder así modificar su texto en todo aquello que se juzgue necesario por la misma Conferencia o que se imponga de acuerdo con los adelantos de la ciencia que para entonces se hubieren alcanzado.

Es interesante que, desde un principio, existieran previsiones para la modificación del Código; es decir, nunca se pensó que fuera inalterable.

Los Gobiernos de México, Panamá y Uruguay ratificaron el Código en el período de 1928 a 1929 y los de Bolivia, Brasil, El Salvador, la República Dominicana y Venezuela en 1930, llegándose a 15 países ese año. En 1931, la República Argentina puso en efecto el Código por Decreto Ejecutivo y la Comisión del Congreso Nacional de Guatemala rindió informe favorable a su ratificación.

El 30 de junio de 1936 el director de la Oficina Sanitaria Panamericana, doctor Hugh S. Cumming, anunció que el tratado internacional denominado Código Sanitario Panamericano había sido ya ratificado por todas las Repúblicas de América.

Del 21 al 30 de julio de 1940 en La Habana, los Cancilleres de América, en la Segunda Reunión de Consulta entre los Ministros de Relaciones Exteriores de las Repúblicas Americanas, considerando que "los resultados satisfactorios obtenidos [en la cooperación sanitaria interamericana] se debieron a la efectiva cooperación entre varios países, la Oficina Sanitaria Panamericana y la Fundación Rockefeller, de acuerdo con el Tratado Sanitario conocido con el nombre de Código Sanitario Panamericano, que todos los países han ratificado" expresaron:

su satisfacción por los eficientes resultados obtenidos hasta la fecha [. . . ] y recomiendan que la cooperación relacionada con las actividades sanitarias continúen y, hasta donde sea posible, se extiendan con la idea de mejorar más aún las condiciones sanitarias, sociales y económicas que se reconocen como esencialmente interdependientes y tanto nacional como internacionalmente beneficiosas.

En 1952 se le agregaba en La Habana un nuevo protocolo anexo al Código Sanitario Panamericano que textualmente dice así:

Los Representantes de los Gobiernos Signatarios del Código Sanitario Panamericano, debidamente autorizados mediante los plenos poderes que les han sido otorgados y que se han encontrado en buena y debida forma; firman en nombre de sus respectivos Gobiernos el presente Protocolo en español, inglés, portugués y francés, en fecha y lugar que aparecen al pie de sus firmas.

\section{ARTÍCULO I}

Se acuerda derogar los Artículos 2, 9, 10, 11, 16 al 53 inclusive, 61 y 62 del Código Sanitario Panamericano, suscrito en La Habana el 14 de noviembre de 1924 durante la VII Conferencia Panamericana, todos los cuales se refieren al tránsito internacional.

\section{ARTÍCULO II}

En adelante cualquier reforma periódica que fuere procedente introducir en los títulos, secciones o artículos del Código Sanitario Panamericano, quedará a cargo de la Conferencia Sanitaria Panamericana; siendo necesario, para que sean válidas las enmiendas, que se aplique en su tramitación lo dispuesto en la Constitución de la Organización Panamericana [denominada actualmente Organización Panamericana de la Salud por decisión de la XV Conferencia Sanitaria Panamericana, septiembre-octubre de 1958].

\section{ARTÍCULO III}

El original del presente Protocolo será depositado en la Unión Panamericana, la cual enviará copias certificadas a los Gobiernos para los fines de ratificación.

\section{ARTÍCULO IV}

El presente Protocolo será ratificado por los Estados Signatarios de acuerdo con sus respectivos procedimientos constitucionales. Los instrumentos de ratificación serán depositados en la Unión Panamericana y esta notificará dicho depósito a los Gobiernos Signatarios.

\section{ARTÍCULO V}

Este Protocolo entrará en vigencia el día primero de octubre de 1952 para aquellos Estados que ratifiquen este instrumento antes de la citada fecha. Para los demás Estados entrará en vigencia a partir de la fecha en que los ratifiquen.

Hecho en la Ciudad de La Habana a los veinticuatro días del mes de septiembre de mil novecientos cincuenta y dos.

Con estas últimas enmiendas se ha mantenido hasta la actualidad el Código Sanitario Panamericano.

\section{PROYECCIONES FUTURAS}

En una Reunión Especial, celebrada en Buenos Aires del 14 al 18 de octubre de 1968, los Ministros de Salud de las Américas propusieron un plan de operaciones para la ejecución de las decisiones 
tomadas por los Presidentes de las Américas en Punta del Este en 1967. Entre los puntos principales, los Ministros subrayaron la legislación sanitaria, incluida la recomendación de que: "Se efectúe, urgentemente, un estudio detenido del Código Sanitario Panamericano, bajo la dirección de la OSP, a la luz del progreso tecnológico, los problemas actuales, y las proyecciones del desarrollo".

El Código, aún en vigencia hoy, es un producto de la época en que apareció y abarca lo que hoy en día podría considerarse un espectro reducido de temas, los cuales eran, en aquel entonces, los problemas de salud preponderantes: enfermedades contagiosas, saneamiento de los puertos y cuaren- tena. Es decir, la situación de salud en el continente ha cambiado, y con el cambio se han vuelto obsoletas muchas de las medidas originalmente prescritas en el Código. Lo que sí queda inmutable es el consenso de los países en cuanto a la necesidad de una institución que sirva de agente para el intercambio de información sanitaria y que abogue por un enfoque panamericano para la solución de problemas de salud comunes. Tal fue la visión que informó el establecimiento de la Oficina Sanitaria Panamericana en 1902. Tal fue el intento del Código Sanitario Panamericano firmado en 1924. Tal es la razón de ser - ahora y siempre- de la Organización Panamericana de la Salud: pro salute Novi Mundi.

\section{BIBLIOGRAFÍA}

Actas de la Séptima Conferencia Sanitaria Panamericana de las Repúblicas Americanas. En: Oficina Sanitaria Panamericana. Actas de la Séptima Conferencia Sanitaria Panamericana de las Repúblicas de América. Washington, D.C.: OSP; 1925:25-121.

Acuña HR. El $75^{\circ}$ Aniversario de la Organización Panamericana de la Salud. Participación de la comunidad en la salud. Educ Med Salud (Washington, D.C.) 1977;11(1):1-2.

Acuña HR. Juventud a los 75. Educ Med Salud (Washington, D.C.) 1977;11(3):200-201.

Anuario Diplomático y Consular de la República de Cuba. Año 1938. La Habana: Imprenta y Papelería de Rambla, Bouza y Cía; 1939.

Balaguer E, Ballester R. Medicina y sociedad. La enfermedad y su prevención. En: Laín Entralgo P. Historia universal de la medicina. Vol. VI. Barcelona: Salvat Editores; 1974:363-370.

Bustamante ME. Los primeros cincuenta años de la Oficina Sanitaria Panamericana. Bol Oficina Sanit Panam 1952;33(6):471-531.

Céspedes y de Quesada CM. de. Discurso en la inauguración de la Séptima Conferencia Sanitaria Panamericana. En: Oficina Sanitaria Panamericana. Actas de la Séptima Conferencia Sanitaria Panamericana de las Repúblicas de América. Washington, D.C.: OSP; 1925: 29-38.

Código Sanitario Panamericano. En: Oficina Sanitaria Panamericana. Actas de la Séptima Conferencia Sanitaria Panamericana de las Repúblicas de América. Washington, D.C.: OSP; 1925:136-167.

Delegados a la Séptima Conferencia Panamericana, 1924. Bol Oficial Secretaria Sanidad Beneficiencia (La Habana) 1924;29:605-661.

Delgado García G. El Código Sanitario Panamericano: aspectos históricos. Cuad Historia Salud Publica (La Habana) 1995:22-35.

Estrella E. Medicina y estructura socioeconómica. Quito: Editorial Belén; 1980.

Estrella E. Historia de la malaria en el Ecuador. En: Kroeger A. et al., eds. Malaria y leishma- niasis cutánea en el Ecuador. Quito: Editorial Abya-Yala; 1991:89-136.

Estrella E. La presencia de la Organización Panamericana de la Salud en la salud pública continental en el siglo XX y la importancia histórica del Código Sanitario Panamericano. Cuad Historia Salud Publica (La Habana) 1995:36-48.

Finlay C. Extracto de las deliberaciones de la Conferencia Sanitaria Internacional de Washington. Obras completas. Tomo I. La Habana: Academia de Ciencias de Cuba; 1965: 199-228.

Finlay C. El mosquito hipotéticamente considerado como agente de transmisión de la fiebre amarilla. En: Finlay C. Obras completas. Tomo I. La Habana: Academia de Ciencias de Cuba; 1965:247-261.

Foucault M. Historia de la medicalización. Educ Med Salud (Washington, D.C.) 1977;11(1): $3-25$.

García-Lebredo Arango M. Discurso en la inauguración de la VII Conferencia Sanitaria Panamericana. En: Oficina Sanitaria Panamericana. Actas de la Séptima Conferencia Sanitaria Panamericana de las Repúblicas de América. Washington, D.C.: OSP; 1925:59-65.

Informes de las Delegaciones a la Séptima Conferencia Sanitaria Panamericana. En: Oficina Sanitaria Panamericana. Actas de la Séptima Conferencia Sanitaria Panamericana de las Repúblicas de América. Washington, D.C.: OSP; 1925:168-280.

La Séptima Conferencia Sanitaria Panamericana. Boletines (1-3) Secretaria Sanidad Beneficencia (La Habana) 1924;29:597-604.

Martí Pérez J. Conferencia Internacional Americana. Obras completas. Tomo 6. La Habana: Editorial Nacional de Cuba; 1963:29-130.

Organización Mundial de la Salud. Evolución de la salud pública internacional. En: Organización Mundial de la Salud. Los diez primeros años de la Organización Mundial de la Salud.
Palacio de las Naciones. Ginebra: OMS; 1958:1-34.

Organización Panamericana de la Salud. Código Sanitario Panamericano. Bol Oficina Sanit Panam 1925;4(2):43-67.

Organización Panamericana de la Salud. ¿Qué es el Código Sanitario Panamericano? Bol Oficina Sanit Panam 1929;8(9):967-968.

Organización Panamericana de la Salud. La Organización Panamericana de la Salud: 75 años de servicio (nota editorial). Educ Med Salud (Washington) 1977;11(4):339-45.

Organización Panamericana de la Salud. Documentos Básicos de la Organización Panamericana de la Salud. Decimocuarta edición. Washington, D.C.: OPS; 1983:128.

Organización Panamericana de la Salud. Pro Salute Novi Mundi: Historia de la Organización Panamericana de la Salud. Washington, D.C.: OPS; 1992:296.

Servicio de Salud Pública de los Estados Unidos de América. Grupo de Archivos 90. College Park, Maryland: Archivos Nacionales de los Estados Unidos de América.

Reglamento de la Séptima Conferencia Sanitaria Panamericana. En: Oficina Sanitaria Panamericana. Actas de la Séptima Conferencia Sanitaria Panamericana de las Repúblicas de América. Washington, D.C.: OSP; 1925:19-24.

Rodríguez Expósito, Finlay C. En: Finlay C. Obras Completas. La Habana: Academia de Ciencias de Cuba, Museo Histórico de las Ciencias Médicas "Carlos J. Finlay" 1965;(1):19-84.

Williams RC. The United States Public Health Service, 1798-1950. Washington, D.C.: Commissioned Officers' Association of the United States Public Health Service; 1951: 443-461.

Zayas AA. Discurso de clausura de la Séptima Conferencia Sanitaria Panamericana. En: Oficina Sanitaria Panamericana. Actas de la Séptima Conferencia Sanitaria Panamericana de las Repúblicas de América. Washington, D.C.: OSP; 1925:118-121. 